\title{
Evaluation of Nano- $\mathrm{Fe}_{3} \mathrm{O}_{4}$ as a Green Catalyst for the Synthesis of Mono, bis and tris Diindolyl Methanes
}

\author{
LEILA ZARE ${ }^{* 1,2}$, AND MOHAMMAD NIKPASSAND ${ }^{2,3}$ \\ ${ }^{1}$ Rasht Branch, Islamic Azad University, Young Researcher Club, Rasht, Iran \\ ${ }^{2}$ Department of Chemistry, Rasht Branch, Islamic Azad University, Rasht, Iran \\ ${ }^{3}$ Department of Chemistry, Payame Noor University, PO Box 19395-3697 Tehran, Iran \\ chem_zare@yahoo.com
}

Received 31 October 2011; Accepted 01 February 2012

\begin{abstract}
Fast and clean synthesis of mono, bis and tris diindolylmethanes from various aldehydes and indoles in the presence of an efficient reusable catalyst, nano- $\mathrm{Fe}_{3} \mathrm{O}_{4}$, in high yield and short reaction time is reported.
\end{abstract}

Keywords: Diindolylmethanes, reusable, nano- $\mathrm{Fe}_{3} \mathrm{O}_{4}$.

\section{Introduction}

It is well known that diindolylmethanes (DIMs) and related compounds exhibit a wide range of biological activities such as antibacterial and antitumor properties ${ }^{1}$. Diindolylmethanes are the most active cruciferous substances for promoting beneficial estrogen metabolism in women and men ${ }^{2}$. DIMs increase the body's natural metabolism of hormones and promote good estrogen (2-hydroxyestrogen). This indole antioxidant is patented for alleviating syptoms of fibromyalgia ${ }^{3}$. Thus indol and its derivatives have been a topic for the preparation of research interest. Several synthetic methods for the preparation of DIMs have been reported by using catalysts such as lanthanide triflate ${ }^{4}, \mathrm{FeCl}_{3}{ }^{5}, \mathrm{HOAc}{ }^{6}$, molecular iodine ${ }^{7}$, montmorillonite clay K-10 ${ }^{8,9}$, indium trichloride and triflate ${ }^{10}$, trifluoroacetic acid ${ }^{11}$, aluminium chloride ${ }^{12}$, ion exchange resin ${ }^{13}$, lithium perchlorate ${ }^{14}$, copper (II) bromide ${ }^{15}$, molibdatophosphoric acid ${ }^{16}$, scandium (III) trifluoromethanesulfonate ${ }^{17}$, zirconium (IV) chloride ${ }^{18}$, bentonite ${ }^{19}$, lewis acids in ionic liquids ${ }^{20}$, ceric ammonium nitrate ${ }^{21}$, silica supported sodium hydrogen sulphate and amberlyst ${ }^{22}$, diphosphooctadecatungstic acid ${ }^{23}$ and $\mathrm{Zr}^{+4}$-dodecyl sulfates ${ }^{24}$. Most of these methods suffer from various disadvantages such as long reaction time, use of expensive lewis acids, toxic metal ions, and harsh reaction condition, excess of toxic or organic solvents, low yield and cumbersome product isolation procedure. Due to these problems, development of an efficient and versatile method for the preparation of DIMs is an important aspect and there is a scope for the further improvement towards milder reaction conditions, improved yields and green procedure. 
In continuation of our ongoing studies to synthesize of heterocyclic and pharmaceutical compounds at mild and practical protocols [25-27], Herein, we wish to report our preliminary results on the preparation of nano- $\mathrm{Fe}_{3} \mathrm{O}_{4}$ (Figure 1) and their application as a green catalyst for the synthesis of mono, bis and tris diindolylmethanes, as shown in (Scheme 1).

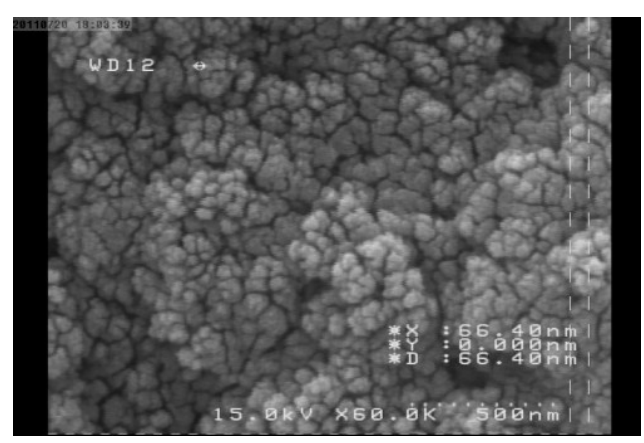

Figure 1. SEM image of synthesized nano- $\mathrm{Fe}_{3} \mathrm{O}_{4}$.

\section{Experimental}

Melting points were measured on an Electrothermal 9100 apparatus. IR spectra were determined on a Shimadzo IR-470 spectrometer. ${ }^{1} \mathrm{H}$ NMR and ${ }^{13} \mathrm{C}$ NMR spectra were recorded on a $500 \mathrm{MHz}$ and $125 \mathrm{MHz}$ Bruker DRX-500 in $\mathrm{CDCl}_{3}$ as solvent and TMS as internal standard. Chemicals were purchased from Merck and Fluka. Elemental analyses were done on a Carlo-Erba EA1110CNNO-S analyzer and agreed with the calculated values. All solvents used were dried and distilled according to standard procedures.

General procedure for the synthesis of $3 a-w$ in the presence of nano- $\mathrm{Fe}_{3} \mathrm{O}_{4}$

A mixture of aldehyde 1 (1 mmol) (scheme 2, entries 3m-u, $0.5 \mathrm{mmol}$ and scheme 3 , entry $3 \mathbf{v}-\mathbf{w}, 0.3 \mathrm{mmol})$, indole derivatives $2(2 \mathrm{mmol})$ and catalytic amount of nano- $\mathrm{Fe}_{3} \mathrm{O}_{4}$ in $\mathrm{H}_{2} \mathrm{O}$ $(10 \mathrm{~mL})$ were stirred for the required reaction times $(5-10 \mathrm{~min})$ at room temperature. The progress of the reaction was monitored by TLC (EtOAc: petroleum ether 1:4). After completion of the reaction, the mixture was filtered in the presence of an efficient magnetic bar. The crude product was recrystallized from ethanol to produce diindolylmethanes derivatives 3a-w as pure crystalline products in $90-98 \%$ yields.

\section{1,4-bis((2-(di(1H-indol-3-yl)methyl)phenoxy)methyl)benzene (3m):}

IR $(\mathrm{KBr}) v=3413,3053,2923,1593,1555,1485,1238 \mathrm{~cm}^{-1} ;{ }^{1} \mathrm{H}$ NMR $(500 \mathrm{MHz}$, DMSO): $\delta=7.69(\mathrm{~s}, 2 \mathrm{H}), 7.4(\mathrm{~d}, J=7.8 \mathrm{~Hz}, 2 \mathrm{H}), 7.2(\mathrm{~d}, J=8.1 \mathrm{~Hz}, 2 \mathrm{H}), 7.16-7.18(\mathrm{~m}, 2 \mathrm{H})$, $7.0(\mathrm{t}, J=7.9 \mathrm{~Hz}, 3 \mathrm{H}), 6.92(\mathrm{~s}, 2 \mathrm{H}), 6.89(\mathrm{t}, 1 \mathrm{H}), 6.57(\mathrm{~d}, J=1.6 \mathrm{~Hz}, 2 \mathrm{H}), 6.39(\mathrm{~s}, 1 \mathrm{H}), 5.0$ (s, 2H) ppm; ${ }^{13} \mathrm{C}$ NMR $(125 \mathrm{MHz}, \mathrm{DMSO}): \delta=147.6,138.5,136.9,133.2,133.1,128.8$, $127.7,126.7,124.4,124.2,123.0,121.2,119.3,118.5,116.5,111.6,70.0,37.9$ ppm; Anal.calcd for $\mathrm{C}_{54} \mathrm{H}_{42} \mathrm{~N}_{4} \mathrm{O}_{2}:$ C, 83.26; H, 5.43; N, 7.19. Found: C, 83.56; H, 5.51; N, 7.09.

\section{1,4-bis((2-(bis(2-methyl-1H-indol-3-yl)methyl)phenoxy)methyl)benzene (3n):}

IR $(\mathrm{KBr}) v=3400,3051,3024,2914,1589,1520,1483,1217 \mathrm{~cm}^{-1} ;{ }^{1} \mathrm{H}$ NMR $(500 \mathrm{MHz}$, DMSO): $\delta=10.68(\mathrm{~s}, 2 \mathrm{H}), 7.20-7.21(\mathrm{~m}, 3 \mathrm{H}), 7.01-7.06(\mathrm{~m}, 2 \mathrm{H}), 6.88(\mathrm{t}, J=7.4 \mathrm{~Hz}, 2 \mathrm{H})$, 6.81-6.84 (m, 5H), $6.66(\mathrm{t}, J=7.5 \mathrm{~Hz}, 2 \mathrm{H}), 6.13(\mathrm{~s}, 1 \mathrm{H}), 4.91(\mathrm{~s}, 2 \mathrm{H}), 1.99(\mathrm{~s}, 6 \mathrm{H}) \mathrm{ppm}$; Anal.calcd for $\mathrm{C}_{58} \mathrm{H}_{50} \mathrm{~N}_{4} \mathrm{O}_{2}$ : C, 83.42; H, 6.04; N, 6.71. Found: C, 83.51; H, 6m bt.34; N, 6.57 . 


\section{1,4-bis((4-(di(1H-indol-3-yl)methyl)phenoxy)methyl)benzene (3o):}

IR $(\mathrm{KBr}) v=3413,3053,2923,1608,1541,1506,1456,1234 \mathrm{~cm}^{-1} ;{ }^{1} \mathrm{H}$ NMR $(500 \mathrm{MHz}$, DMSO): $\delta=10.70(\mathrm{~s}, 2 \mathrm{H}), 7.43(\mathrm{~s}, 2 \mathrm{H}), 7.34(\mathrm{~d}, J=8.1 \mathrm{~Hz}, 2 \mathrm{H}), 7.25-7.28(\mathrm{~m}, 3 \mathrm{H}), 7.03(\mathrm{t}$, $J=7.7 \mathrm{~Hz}, 2 \mathrm{H}), 6.91(\mathrm{~d}, J=8.6 \mathrm{~Hz}, 2 \mathrm{H}), 6.85(\mathrm{t}, J=7.6 \mathrm{~Hz}, 2 \mathrm{H}), 6.80(\mathrm{~d}, J=2 \mathrm{~Hz}, 3 \mathrm{H}), 5.77$ (s, $1 \mathrm{H}), 5.0$ ( s, 2H) ppm; Anal.calcd for $\mathrm{C}_{54} \mathrm{H}_{42} \mathrm{~N}_{4} \mathrm{O}_{2}: \mathrm{C}, 83.26 ; \mathrm{H}, 5.43 ; \mathrm{N}, 7.19$. Found: $\mathrm{C}$, 83.41; H, 5.68; N, 7.09.

\section{1,4-bis((4-(bis(2-methyl-1H-indol-3-yl)methyl)phenoxy)methyl)benzene (3p):}

IR $(\mathrm{KBr}) v=3409,3055,2916,1602,1540,1460,1298 \mathrm{~cm}^{-1} ;{ }^{1} \mathrm{H}$ NMR $(500 \mathrm{MHz}$, DMSO): $\delta=10.71(\mathrm{~s}, 2 \mathrm{H}), 7.45(\mathrm{~s}, 2 \mathrm{H}), 7.2(\mathrm{~d}, J=7.9 \mathrm{~Hz}, 2 \mathrm{H}), 7.0(\mathrm{~d}, J=8.5 \mathrm{~Hz}, 2 \mathrm{H})$, 6.87- $6.91(\mathrm{~m}, 4 \mathrm{H}), 6.82(\mathrm{~d}, J=7.9 \mathrm{~Hz}, 2 \mathrm{H}), 6.68(\mathrm{t}, J=7.6 \mathrm{~Hz}, 2 \mathrm{H}), 5.86(\mathrm{~s}, 1 \mathrm{H}), 5.07(\mathrm{~s}$, 2H), $2.06(\mathrm{~s}, 6 \mathrm{H})$ ppm. Anal.calcd for $\mathrm{C}_{58} \mathrm{H}_{50} \mathrm{~N}_{4} \mathrm{O}_{2}: \mathrm{C}, 83.42 ; \mathrm{H}, 6.04 ; \mathrm{N}, 6.71$. Found: $\mathrm{C}$, $83.51 ; \mathrm{H}, 6.34 ; \mathrm{N}, 6.57$.

\section{1,4-bis((4-(bis(1-methyl-1H-indol-3-yl)methyl)phenoxy)methyl)benzene (3q):}

IR $(\mathrm{KBr}) v=3053,2925,1595,1550,1481,1458,1240 \mathrm{~cm}^{-1} ;{ }^{1} \mathrm{H}$ NMR $(500 \mathrm{MHz}$, DMSO): $\delta=7.42(\mathrm{~d}, J=7.9 \mathrm{~Hz}, 2 \mathrm{H}), 7.30(\mathrm{t}, J=8.1 \mathrm{~Hz}, 2 \mathrm{H}), 7.19-7.26(\mathrm{~m}, 4 \mathrm{H}), 6.99-7.04$ $(\mathrm{m}, 4 \mathrm{H}), 6.89(\mathrm{~d}, J=7.9 \mathrm{~Hz}, 2 \mathrm{H}), 6.58(\mathrm{~s}, 2 \mathrm{H}), 5.17(\mathrm{~s}, 1 \mathrm{H}), 5.06(\mathrm{~s}, 2 \mathrm{H}), 3.67$ (s, 6H) ppm; ${ }^{13} \mathrm{C}$ NMR (125 MHz, DMSO): $\delta=156.31,137.80,136.36,133.89,133.86,128.8,128.7$, 127.5, 120.5, 118,3, 113.4, 112.6, 109.4, 70.1, 66.3, 33.6 ppm; Anal.calcd for $\mathrm{C}_{58} \mathrm{H}_{50} \mathrm{~N}_{4} \mathrm{O}_{2}$ : C, 83.42; H, 6.04; N, 6.71. Found: C, 83.61; H, 6.57; N, 6.09.

\section{1,5-bis(2-(di(1H-indol-3-yl)methyl)phenoxy)pentane (3r):}

IR $(\mathrm{KBr}) v=3460,3406,2927,1631,1452,1245 \mathrm{~cm}^{-1} ;{ }^{1} \mathrm{H}$ NMR $(500 \mathrm{MHz}, \mathrm{DMSO}): \delta=$ $10.7(\mathrm{~s}, 2 \mathrm{H}), 7.36(\mathrm{~d}, J=8.0 \mathrm{~Hz}, 2 \mathrm{H}), 7.28(\mathrm{~d}, J=7.9 \mathrm{~Hz}, 2 \mathrm{H}), 7.14(\mathrm{t}, J=7.62 \mathrm{~Hz}, 2 \mathrm{H})$, $7.02(\mathrm{t}, J=8.0 \mathrm{~Hz}, 2 \mathrm{H}), 6.89(\mathrm{~d}, J=8.1 \mathrm{~Hz}, 1 \mathrm{H}), 6.85(\mathrm{t}, J=7.3 \mathrm{~Hz}, 2 \mathrm{H}), 6.80(\mathrm{t}, J=7.4 \mathrm{~Hz}$, $3 \mathrm{H}), 6.20(\mathrm{~s}, 1 \mathrm{H}), 3.77(\mathrm{t}, J=6.0 \mathrm{~Hz}, 2 \mathrm{H}), 1.54(\mathrm{~m}, 2 \mathrm{H}), 1.24(\mathrm{~m}, 1 \mathrm{H}) \mathrm{ppm} ;{ }^{13} \mathrm{C}$ NMR $(125$ MHz, DMSO): $\delta=156.56,137.43,133.98,129.83,127.74,127.68,124.48,121.64$, $120.75,119.70,118.95,118.80,112.67,112.26,68.36,32.43,29.29,22.95$ ppm; Anal.calcd for $\mathrm{C}_{51} \mathrm{H}_{44} \mathrm{~N}_{4} \mathrm{O}_{2}:$ C, 82.23; H, 5.95; N, 7.52;. Found: C, 82.01; H, 5.68; N, 7.83.

\section{1,5-bis(2-(bis(2-methyl-1H-indol-3-yl)methyl)phenoxy)pentane (3s):}

IR $(\mathrm{KBr}) v=3469,3413,2925,1625,1460,1232 \mathrm{~cm}^{-1} ;{ }^{1} \mathrm{H}$ NMR (500 MHz, DMSO): $\delta=$ $10.65(\mathrm{~s}, 2 \mathrm{H}), 7.16(\mathrm{~d}, J=7.5 \mathrm{~Hz}, 2 \mathrm{H}), 7.00(\mathrm{~d}, J=7.0 \mathrm{~Hz}, 2 \mathrm{H}), 6.80-6.82(\mathrm{~m}, 3 \mathrm{H}), 6.75-$ $6.78(\mathrm{~m}, 3 \mathrm{H}), 6.61(\mathrm{t}, J=7.1 \mathrm{~Hz}, 2 \mathrm{H}), 6.02(\mathrm{~s}, 1 \mathrm{H}), 3.48(\mathrm{br}, 2 \mathrm{H}), 2.01(\mathrm{br}, 2 \mathrm{H}), 1.96(\mathrm{~s}$, $6 \mathrm{H}), 1.11(\mathrm{~m}, 1 \mathrm{H}) \mathrm{ppm}$; Anal.calcd for $\mathrm{C}_{55} \mathrm{H}_{52} \mathrm{~N}_{4} \mathrm{O}_{2}: \mathrm{C}, 82.47 ; \mathrm{H}, 6.54 ; \mathrm{N}, 6.99$. Found: $\mathrm{C}$, 82.64; H, 6.33; N, 6.73.

\section{1,6-bis(2-(di(1H-indol-3-yl)methyl)phenoxy)hexane (3t):}

IR $(\mathrm{KBr}) v=3409,3055,2916,1602,1540,1460,1298 \mathrm{~cm}^{-1} ;{ }^{1} \mathrm{H}$ NMR (500 MHz, DMSO): $\delta=10.7(\mathrm{~s}, 2 \mathrm{H}), 7.33(\mathrm{~d}, J=8.0 \mathrm{~Hz}, 2 \mathrm{H}), 7.26(\mathrm{~d}, J=7.9 \mathrm{~Hz}, 2 \mathrm{H}), 7.10-7.14(\mathrm{~m}, 2 \mathrm{H}), 6.99$ $(\mathrm{t}, J=7.76 \mathrm{~Hz}, 2 \mathrm{H}), 6.92(\mathrm{~d}, J=8.01 \mathrm{~Hz}, 1 \mathrm{H}), 6.83(\mathrm{t}, J=7.6 \mathrm{~Hz}, 2 \mathrm{H}), 6.79(\mathrm{t}, J=7.5 \mathrm{~Hz}$, $1 \mathrm{H}), 6.76(\mathrm{~d}, J=1.67 \mathrm{~Hz}, 2 \mathrm{H}), 6.01(\mathrm{~s}, 1 \mathrm{H}), 3.86(\mathrm{t}, J=6.0 \mathrm{~Hz}, 2 \mathrm{H}), 1.47(\mathrm{br}, 2 \mathrm{H}), 1.19(\mathrm{br}$, 2H) ppm; ${ }^{13} \mathrm{C}$ NMR $(125 \mathrm{MHz}, \mathrm{DMSO}): \delta=156.56,137.41,134.03,129.88,127.74$, $127.73,127.65,121.62,120.75,119.64,118.92,118.82,112.67,112.23,68.44,32.28$, 29.62, 26.13 ppm; Anal.calcd for $\mathrm{C}_{52} \mathrm{H}_{46} \mathrm{~N}_{4} \mathrm{O}_{2}$ : C, 82.29; H, 6.11; N, 7.38. Found: C, 82.27; H, 6.33; N, 7.81 .

\section{1,4-bis(4-(bis(2-methyl-1H-indol-3-yl)methyl)-2-methoxyphenoxy)butane (3u):}

IR $(\mathrm{KBr}) v=3420,3085,2934,1602,1560,1467,1460,1298 \mathrm{~cm}^{-1} ;{ }^{1} \mathrm{H}$ NMR $(500 \mathrm{MHz}$, DMSO): $\delta=10.7(\mathrm{~s}, 2 \mathrm{H}), 7.21(\mathrm{~d}, J=8.0 \mathrm{~Hz}, 2 \mathrm{H}), 6.88(\mathrm{~m}, 5 \mathrm{H}), 6.82(\mathrm{td}, J=8.3 \mathrm{~Hz}, 1 \mathrm{H})$, 
$6.69(\mathrm{t}, J=7.21 \mathrm{~Hz}, 2 \mathrm{H}), 6.62(\mathrm{dd}, J=8.3 \mathrm{~Hz}, \mathrm{~J}=1.3 \mathrm{~Hz}, 2 \mathrm{H}), 5.86(\mathrm{~s}, 1 \mathrm{H}), 3.86(\mathrm{t}, J=$ $6.0 \mathrm{~Hz}, 2 \mathrm{H}), 3.98(\mathrm{br}, 2 \mathrm{H}), 3.56(\mathrm{~s}, 3 \mathrm{H}), 2.07(\mathrm{~s}, 6 \mathrm{H}), 1.85(\mathrm{br}, 2 \mathrm{H}) \mathrm{ppm} ;{ }^{13} \mathrm{C}$ NMR $(125$ MHz, DMSO): $\delta=149.59,147.21,137.60,132.75,129.18,121.44,120.35,119.35,118.73$, $114.25,113.57,113.40,111.13,68.85,56.38,26.52,12.82 \mathrm{ppm}$; Anal.calcd for $\mathrm{C}_{56} \mathrm{H}_{54} \mathrm{~N}_{4} \mathrm{O}_{4}$ : C, 79.40; H, 6.43; N, 6.61. Found: C, 79.22; H, 6.27; N, 6.91.

\section{1,3,5-tris((4-(di(1H-indol-3-yl)methyl)phenoxy)methyl)benzene (3v):}

Light red solid, IR (KBr) $v=3431,3100,2980,1635,1506,1480,1387,1206,1173 \mathrm{~cm}^{-1}$; ${ }^{1} \mathrm{H}$ NMR $\left(500 \mathrm{MHz}, \mathrm{DMSO}-\mathrm{d}_{6}\right): \delta=10.71(\mathrm{~s}, 6 \mathrm{H}), 6.74-7.50(\mathrm{~m}, 45 \mathrm{H}), 5.72(\mathrm{~s}, 3 \mathrm{H}), 5.01$ $(\mathrm{s}, 6 \mathrm{H}) \mathrm{ppm} ;{ }^{13} \mathrm{C}$ NMR $\left(250 \mathrm{MHz}, \mathrm{DMSO}-\mathrm{d}_{6}\right): \delta=40.08,69.48,111.85,114.67,115.24$, $118.54,118.82,119.58,121.25,123.87,127.05,129.66,137.03,137.73,138.15$, 156.91ppm; Anal. Calc. for $\mathrm{C}_{78} \mathrm{H}_{60} \mathrm{~N}_{6} \mathrm{O}_{3}$ : C, 82.95; H, 5.35; N, 7.44. Found: C, 82.62; H, $5.26 ; \mathrm{N}, 7.91$.

\section{2,4,6-tris(4-(di(1H-indol-3-yl)methyl)phenoxy)-1,3,5-triazine (3w):}

IR $(\mathrm{KBr}) v=3421,3050,2921,1600,1575,1500,1470,1365,1240,1150 \mathrm{~cm}^{-1} ;{ }^{1} \mathrm{H}$ NMR $\left(500 \mathrm{MHz}, \mathrm{DMSO}-\mathrm{d}_{6}\right): \delta=10.8(\mathrm{~s}, 6 \mathrm{H}), 7.85(\mathrm{~m}, 6 \mathrm{H}), 7.35(\mathrm{~m}, 12 \mathrm{H}), 7.32(\mathrm{~d}, J=7.8 \mathrm{~Hz}$, $6 \mathrm{H}), 7.00(\mathrm{~d}, J=8.4 \mathrm{~Hz}, 6 \mathrm{H}), 6.83-6.89(\mathrm{~m}, 12 \mathrm{H}), 5.84(\mathrm{~s}, 3 \mathrm{H}) \mathrm{ppm} ;{ }^{13} \mathrm{CNMR}(250 \mathrm{MHz}$, DMSO-d $\left.{ }_{6}\right): \delta=173.58,149.91,143.45,137.00,129.44,127.52,124.09,121.67,121.45$, 119.76, 119.00, 118.27, 111.90, 30.11 ppm; Anal. Calc. for $\mathrm{C}_{72} \mathrm{H}_{51} \mathrm{~N}_{9} \mathrm{O}_{3}$ : C, 79.32; H, 4.72; N, 11.56. Found: C, 79.32; H, 4.72; N, 11.51.

\section{Results and discussion}

Initially, the condensation reaction of 4-nitrobenzaldehyde and indole in the presence of $\mathrm{Fe}_{3} \mathrm{O}_{4}$ and nano- $\mathrm{Fe}_{3} \mathrm{O}_{4}$ was done and the results were listed in Table 1.

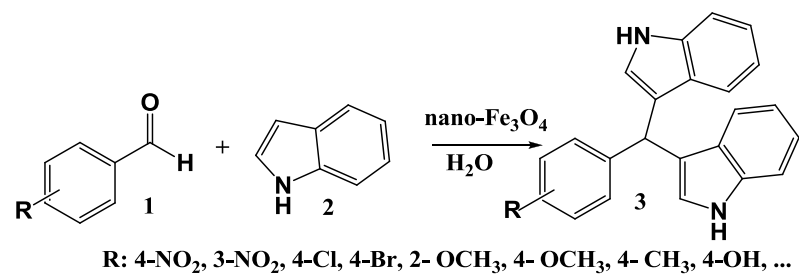

Scheme 1

As depicted, nano- $\mathrm{Fe}_{3} \mathrm{O}_{4}$ proved to be better catalyst than $\mathrm{Fe}_{3} \mathrm{O}_{4}$ for the synthesis of diindolylmethanes. With the best catalyst in hand, we moved to study the effects of catalyst amount on the model reaction and the results were listed in (Table 1). 50mg of nano- $\mathrm{Fe}_{3} \mathrm{O}_{4}$ is sufficient to push the reaction forward completion and $10 \mathrm{mg}$ and $30 \mathrm{mg}$ of catalyst was not enough. Higher amount of catalyst did not lead to significant change in the reaction yields.

Table 1.The effect of amount of nano- $\mathrm{Fe}_{3} \mathrm{O}_{4}$ for the synthesis of $\mathbf{3 a}$.

\begin{tabular}{ccccc}
\hline entry & catalyst & Catalyst amount $(\%)$ & Time $(\min )$ & Yield $(\%)^{\mathrm{a}}$ \\
\hline 1 & - & - & 720 & No reaction \\
\hline 2 & $\mathrm{Fe}_{3} \mathrm{O}_{4}$ & $50 \mathrm{mg}$ & 80 & 65 \\
\hline 3 & $\mathrm{Nano}-\mathrm{Fe}_{3} \mathrm{O}_{4}$ & $50 \mathrm{mg}$ & 5 & 98 \\
\hline 4 & Nano- $\mathrm{Fe}_{3} \mathrm{O}_{4}$ & $10 \mathrm{mg}$ & 30 & 83 \\
\hline 5 & Nano- $\mathrm{Fe}_{3} \mathrm{O}_{4}$ & $30 \mathrm{mg}$ & 15 & 96 \\
\hline 6 & Nano- $\mathrm{Fe}_{3} \mathrm{O}_{4}$ & $100 \mathrm{mg}$ & 5 & 98 \\
\hline
\end{tabular}

a. isolated yield. 
With the best quantity of nano- $\mathrm{Fe}_{3} \mathrm{O}_{4}$ in hand, we were interested to synthesis several diindolylmethanes at room temperature with this catalyst. The results are summarized in Table 2.

Table 2. Synthesis of diindolylmethanes in the presence of nano- $\mathrm{Fe}_{3} \mathrm{O}_{4}$.

Entry aldehydes $\quad M . P\left({ }^{\circ} \mathrm{C}\right)$

\begin{tabular}{|c|c|c|c|c|c|}
\hline & & \multirow{3}{*}{\multicolumn{2}{|c|}{ Yield(\%) }} & \\
\hline & & & & & \\
\hline & & & & Found & Reported \\
\hline $3 a$ & 4- $\mathrm{NO}_{2} \mathrm{C}_{6} \mathrm{H}_{4} \mathrm{CHO}$ & $5(80)^{a}$ & $98(65)^{\mathrm{a}}$ & $235-236$ & $\begin{array}{l}222-228 \\
{[23]}\end{array}$ \\
\hline $3 b$ & $3-\mathrm{NO}_{2} \mathrm{C}_{6} \mathrm{H}_{4} \mathrm{CHO}$ & 5 & 94 & $261-263$ & $\begin{array}{l}265-266 \\
{[23]}\end{array}$ \\
\hline $3 c$ & $\mathrm{C}_{6} \mathrm{H}_{5} \mathrm{CHO}$ & 7 & 85 & $112-113$ & $\begin{array}{l}125-127 \\
{[23]}\end{array}$ \\
\hline $3 \mathrm{~d}$ & $2-\mathrm{Cl} \mathrm{C}{ }_{6} \mathrm{H}_{4} \mathrm{CHO}$ & 10 & 83 & $76-77$ & $72-74[7]$ \\
\hline $3 \mathrm{e}$ & 4- $\mathrm{Cl} \mathrm{C} \mathrm{C}_{6} \mathrm{H}_{4} \mathrm{CHO}$ & 5 & 96 & $80-82$ & $77-81[23]$ \\
\hline $3 \mathrm{f}$ & $2-\mathrm{OCH}_{3} \mathrm{C}_{6} \mathrm{H}_{4} \mathrm{CHO}$ & 7 & 84 & $129-130$ & $\begin{array}{l}134-136 \\
{[23]}\end{array}$ \\
\hline $3 \mathrm{~g}$ & 4- $\mathrm{OCH}_{3} \mathrm{C}_{6} \mathrm{H}_{4} \mathrm{CHO}$ & 10 & 82 & $187-188$ & $\begin{array}{l}\text { 191-193 } \\
{[23]}\end{array}$ \\
\hline $3 \mathrm{~h}$ & 4- $\mathrm{CH}_{3} \mathrm{C}_{6} \mathrm{H}_{4} \mathrm{CHO}$ & 10 & 84 & $92-94$ & $95-97[23]$ \\
\hline $3 \mathrm{i}$ & 4- $\mathrm{OH} \mathrm{C}{ }_{6} \mathrm{H}_{4} \mathrm{CHO}$ & 10 & 87 & $122-124$ & $\begin{array}{l}122-124 \\
{[23]}\end{array}$ \\
\hline $3 \mathrm{j}$ & $\begin{array}{l}\text { Thiophen-2- } \\
\text { carbaldehyde }\end{array}$ & 7 & 86 & $149-150$ & $151-153[7]$ \\
\hline $3 \mathrm{k}$ & Furan-2-carbaldehyde & 7 & 89 & $310-312$ & $322-323[7]$ \\
\hline 31 & Indole -3-carbaldehyde & 5 & 92 & $165-166$ & $160[24]$ \\
\hline
\end{tabular}

\footnotetext{
${ }^{\mathrm{a}}$. A mixture of aldehyde $\mathbf{1 a}(1 \mathrm{mmol})$ and indole derivatives $2(2 \mathrm{mmol})$ and $0.05 \mathrm{~g} \mathrm{Fe}_{3} \mathrm{O}_{4}$ in $10 \mathrm{ml} \mathrm{H}_{2} \mathrm{O}$ were stirred for the required reaction times. ${ }^{\mathrm{b}}$. Isolated yield. ${ }^{\mathrm{c} .}$ The products were characterized by comparison of their spectroscopic and physical data with those of samples synthesized by reported procedures.
}

Interestingly, this reaction due to their reduced pollution, low cost, simplicity in process and handling using nano- $\mathrm{Fe}_{3} \mathrm{O}_{4}$ as catalyst, also afforded an efficient protocol for the synthesis of bis diindolylmethanes (Figure 1, Entry 3m-u) and tris diindolylmethanes (Figure 2, Entry 3v-u) as novel arylmethanes in high yields and short reaction time. The results are summarized in Table 3. 
Table 3. Synthesis of bis and tris diindolylmethanes in the presence of nano- $\mathrm{Fe}_{3} \mathrm{O}_{4}$.

\begin{tabular}{ccccc}
\hline Entry & Product & Time (min) & Yield $(\mathbf{\%})^{\mathbf{a}}$ & m.p. $\left({ }^{\circ} \mathbf{C}\right)$ \\
\hline 1 & $3 \mathrm{~m}$ & 9 & 83 & $212-214$ \\
2 & $3 \mathrm{n}$ & 7 & 81 & $193-194$ \\
3 & $3 \mathrm{o}$ & 7 & 92 & $151-152$ \\
4 & $3 \mathrm{p}$ & 7 & 89 & $206-208$ \\
5 & $3 \mathrm{q}$ & 8 & 88 & $281-283$ \\
6 & $3 \mathrm{r}$ & 7 & 96 & $191-195$ \\
7 & $3 \mathrm{~s}$ & 9 & 93 & $184-185$ \\
8 & $3 \mathrm{t}$ & 8 & 91 & $291-292$ \\
9 & $3 \mathrm{u}$ & 7 & 93 & $284-285$ \\
10 & $3 \mathrm{v}$ & 8 & 91 & $202-204$ \\
11 & $3 \mathrm{w}$ & 9 & 94 & $234-235$ \\
\hline
\end{tabular}

a. Isolated yield.
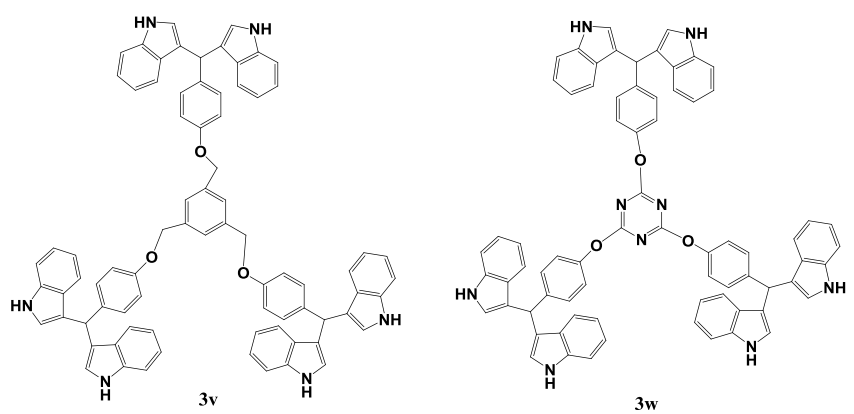

Figure 2
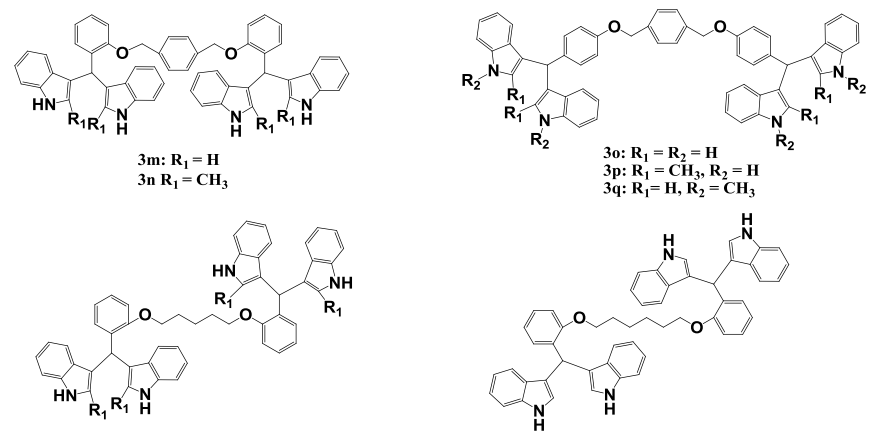

3r: $\mathbf{R}_{1}=\mathbf{H}$
3s: $\mathbf{R}_{1}=\mathbf{C H}_{3}$

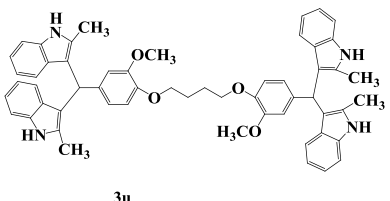

Figure 3 
Apart from the mild condition of the process and its excellent results, the simplicity of product isolation, replacement of carcinogenic solvent with $\mathrm{H}_{2} \mathrm{O}$ and the possibility to recycle nano- $\mathrm{Fe}_{3} \mathrm{O}_{4}$ offer a significant advantage. After the reaction was completed, the catalyst could be easily attracted by an efficient magnetic bar. After further treatments including washing with $\mathrm{CHCl}_{3}$ and activation at $80^{\circ} \mathrm{C}$, the recycled catalyst has been examined in next run. Studies on the synthesis of 3a as model substrate showed that the recovered catalyst could be successively recycled in subsequent reactions without any decrease of yields (Figure 4).

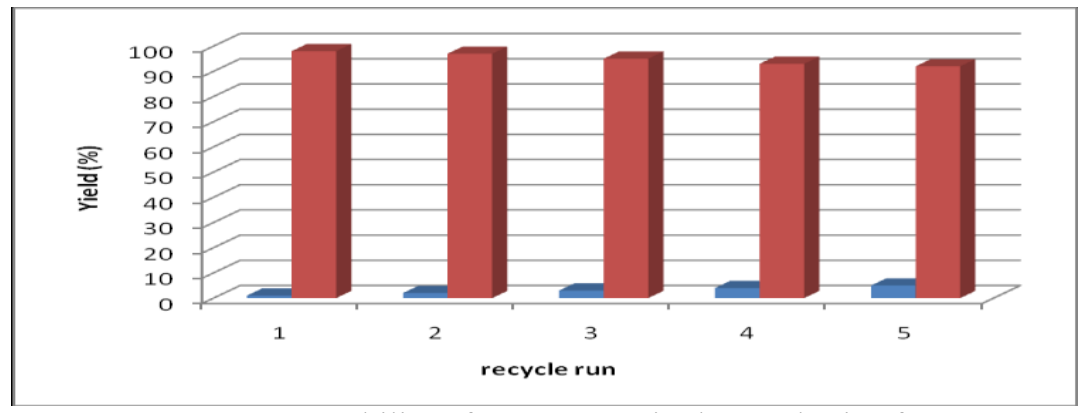

Figure 4. Reusability of nano- $\mathrm{Fe}_{3} \mathrm{O}_{4}$ in the synthesis of 3a.

We propose a possible mechanism for the nano- $\mathrm{Fe}_{3} \mathrm{O}_{4}$ catalyzed synthesis of diindolylmethanes (3) (Scheme 2). We suggest that, nano- $\mathrm{Fe}_{3} \mathrm{O}_{4}$ catalyze the formation of iminium ion (4) in a reversible reaction with the aldehyde. The higher reactivity of the iminium ion compared to the carbonyl species could facilitate nucleophilic attack of indole to aldehyde (1), via intermediate (5) and after the treatment of second molecules of indole and the elimination of nano- $\mathrm{Fe}_{3} \mathrm{O}_{4},(3)$ might be produced.
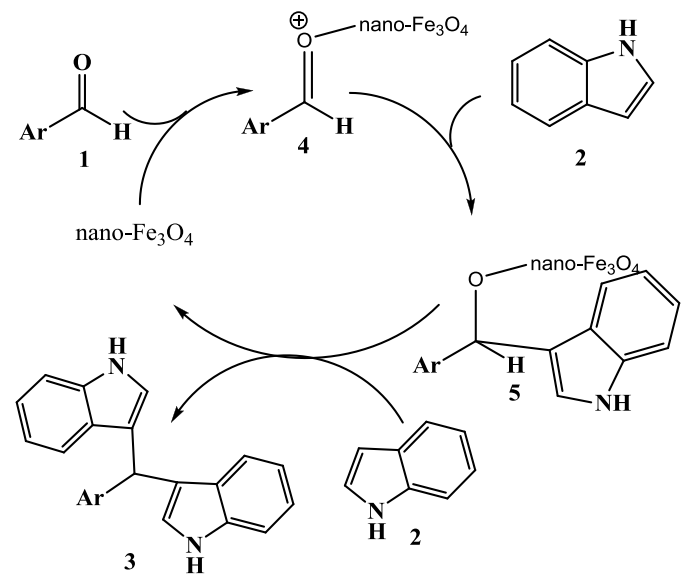

Scheme 2

The selectivity of the present methods is also demonstrated by competitive reactions of indol with arylaldehydes with electron donating and electron withdrawing substitutents. For example, when a 1:1 mixture of 4-methoxybenzaldehyde and 4-nitrobenzaldehyde was allowed to react with two equivalents of indole in the presence of nano- $\mathrm{Fe}_{3} \mathrm{O}_{4}$ under modified conditions, it was found that the arylaldehydes bearing withdrawing substituents 
was chemo selectively converted to the corresponding bis(indolyl)methane but the aryl aldehydes with donating group was converted slightly (Scheme 3).

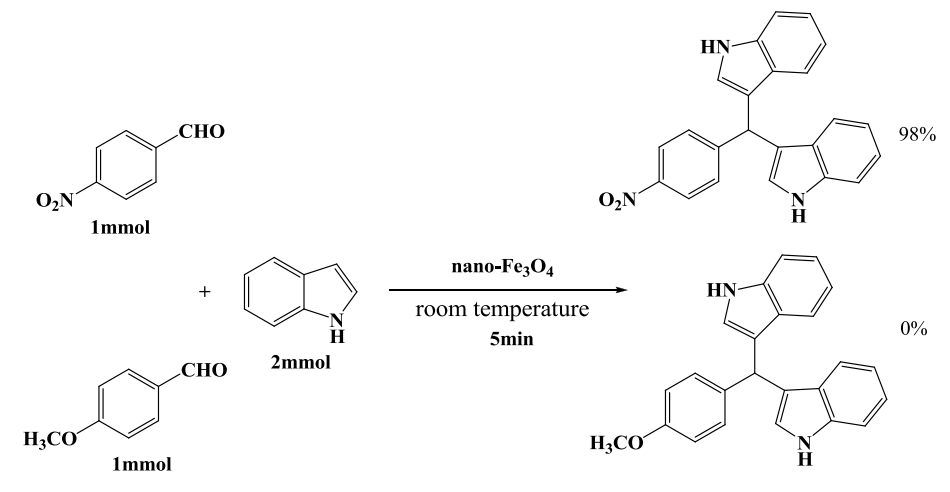

Scheme 3

All of compounds summarized in (Table 2 and Figure 1 and 2) were characterized by spectroscopic methods (IR, ${ }^{1} \mathrm{HNMR}$ and ${ }^{13} \mathrm{CNMR}$ ) and elemental analysis.

\section{Conclusion}

Finally, we develop an efficient and convenient procedure for the synthesis of diindolylmethanes through electrophilic reaction of indoles and aldehydes over nano- $\mathrm{Fe}_{3} \mathrm{O}_{4}$ catalyst. This procedure offer advantages such as reduced reaction time, mild reaction condition, productivity and higher yield, ease of execution and economic viability of the catalyst. This simple process combined with easy of recovery and reuse of catalyst make this procedure economic, begin and a waste free chemical process for the synthesis of diindolylmethanes.

\section{Acknowledgment}

We are pleased to acknowledge the financial support from Young researcher club and Islamic Azad university-Rasht branch.

\section{References}

1. Drasar B S, Hill M J, Human Intestinal Flora., Academic Press, New York, 1974.

2. Zeligs M A, J Med Food., 1998, 1, 67.

3. Michnovicz J J, Bradlowin H L, Huang M J, Osawa T, Ho C T, Rosen R T, Food Phytochemicals for Cancer Prevention 1., Washington, DC, 1994, 282.

4. Chen D, Yu L, Wang P G, Tetrahedron Lett., 1996 ,37, 4467.

5. Vigmond S J, Chang M C, Kallury K M R, Thompson M, Tetrahedron Lett., 1994, 35, 2455.

6. (a) A. Kamal, A. A. Qureshi, Tetrahedron 19 (1963) 513- ; (b) W. Noland, M. Venkiteswaren, C. Richards, J. Org. Chem. 26 (1961) 4241-

7. B.P. Bandgar, K.A. Shaikh, Tetrahedron Lett. 44 (2003) 1959-

8. M. Chakrabarty, N. Ghosh, R. Basak, Y. Harigaya, Tetrahedron Lett. 43 (2002) 4075-

9. A.K. Maiti, P. Bhattacharyya, J. Chem. Res. S (1997) 424- 
10. (a) G. Babu, N. Sridhar, P.T. Perumal, Synth. Commun. 30 (2000) 1609- ; (b) R. Nagarajan, P.T. Perumal, Tetrahedron 58 (2002) 1229 -.

11. C.H. Lee, J.S. Lindsey, Tetrahedron 1994, 50, 11427.

12. A. Chatterjee, S. Manna, T. Prange, J. Shoolery, J. Chem. Soc. Perkin, 1980, 1, 553;

13. X. Feng, C. Guan, C. Zhao, Synth. Commun., 2004, 34, 487.

14. J. S. Yadav, B. S. Reddy, C. Murthy, G. M. Kumar, C. Madan, Synthesis, 2001, 787.

15. L. P. Mo, Z. Ch. Ma, Zh. H. Zhang, Synth. Commun., 2005, 35, 1997.

16. M. A. Zolfigol, P. Salehi, M. Shiri, Phosphorus Sulfur Silicon Rel. Elements, 2004, 178 149.

17. S. Sato, T. Sato, Carbohyd. Res., 2005, 340, 2251.

18. R.R. Nagawade, D. B. Shinde, Bull. Korean Chem. Soc., 2005, 26,1962.

19. G. Penieres-Carrillo, J. G. Garcia-Estrada, J. L. Gutierrez-Ramirez, C. AlvarezToledano, Green Chem., 2003, 5, 337.

20. (a) S. J. Ji, M. F. Zhou, D. G. Gu, S. Y. Wang, T. P. Loh, Synlett, 2003, 2077.

(b) D. G. Gu, S. J. Ji, Z. Q. Jiang, M. F. Zhou, T. P. Loh, Synlett, 2005, 959.

21. C. Ramesh, N. Ravindranath, B. Das, J. Chem. Res., 2003, S, 72.

22. Ch. Ramesh, J. Banerjee, R. Pal, B. Das, Adv. Synth. Catal., 2003, 345, 557.

23. M. Heravi, Kh. Bakhtiari, A. Fatehi, F. Bamoharram, Catal. Commun., 2008, 9 , 289.

24. M. A. Zolfigol, P. Salehi, M. Shiri, Z. Tanbakouchian, Catal. Commun. 2007, 8173.

25. L Zare, M Nikpassand, Chin.Chem.Lett. 2011, 22, 331.

26. M Nikpassand, L Zare, M Saberi, Monatsch. Chem. (in press)

27. M Nikpassand, L Zare, T Shafaati, Chin. J. Chem (in press) 


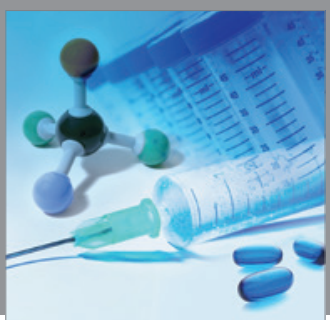

International Journal of

Medicinal Chemistry

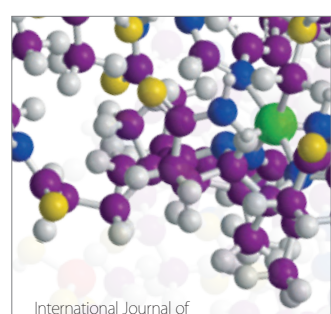

Carbohydrate Chemistry

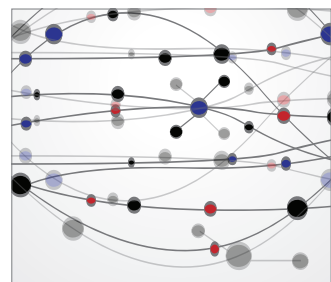

The Scientific World Journal
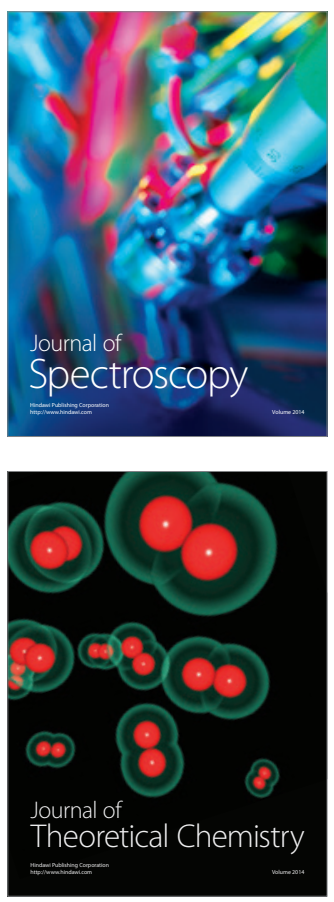
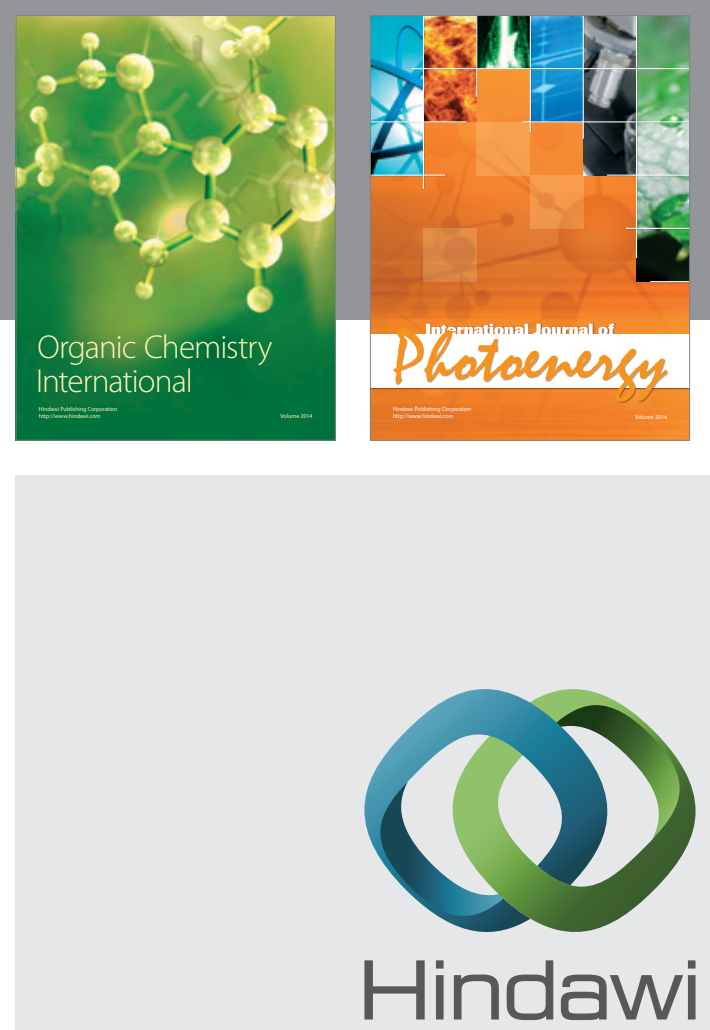

Submit your manuscripts at

http://www.hindawi.com
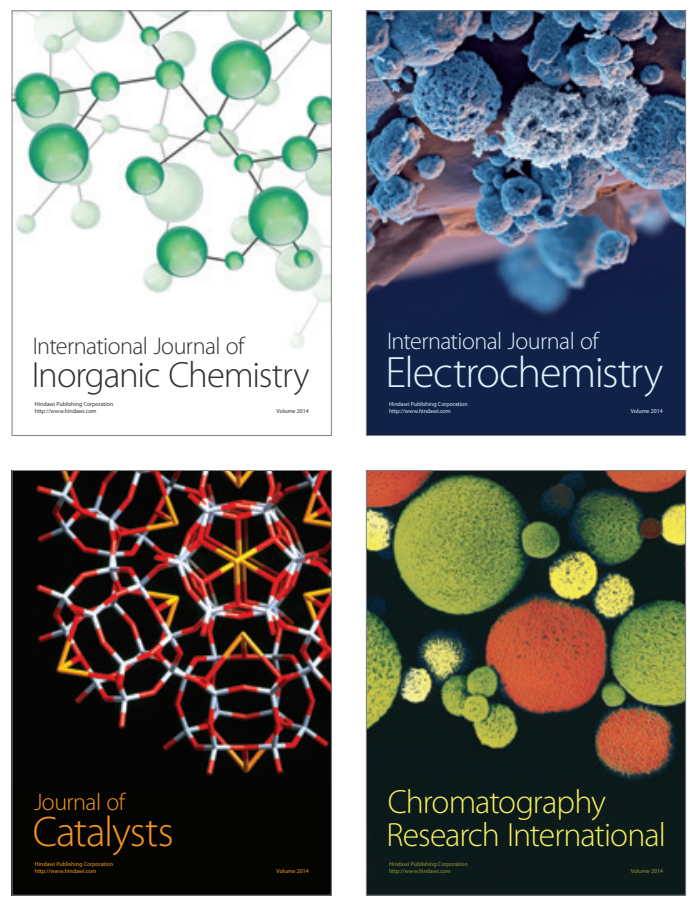
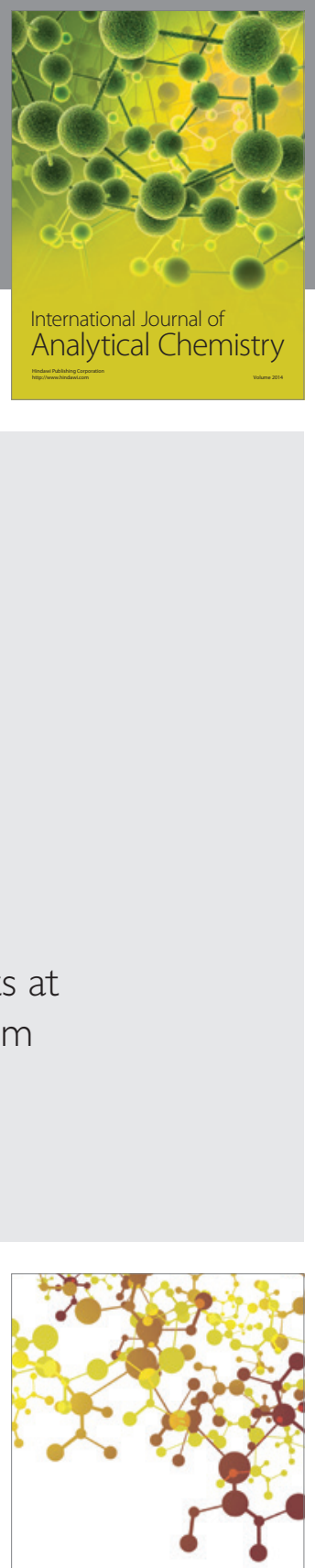

Journal of

Applied Chemistry
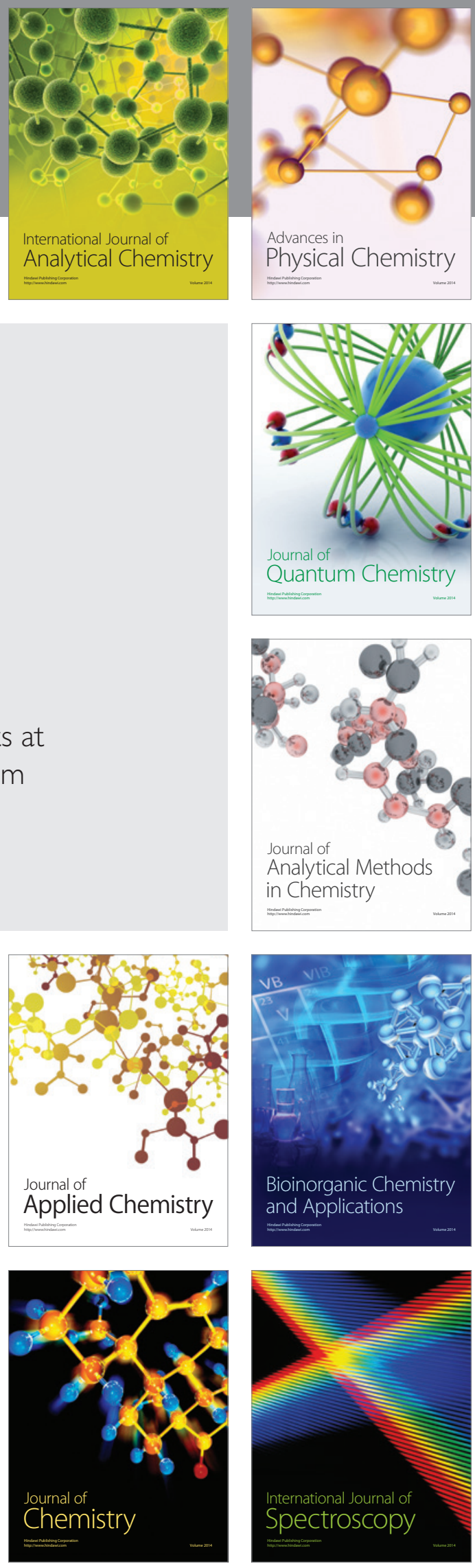